\title{
The X-Ray Jet in the Crab Nebula: Radical Implications for Pulsar Theory?
}

\author{
Yury Lyubarsky, David Eichler \\ Department of Physics, Ben-Gurion University, Beer-Sheva 84105, Israel \\ lyub, eichler@bgumail.bgu.ac.il
}

\begin{abstract}
The recent Chandra image of the Crab nebula shows a striking, axisymmetric polar jet. It is shown that jets are formed in axisymmetric, magnetized pulsar winds and that the jet luminosity scales relative to the total as $\left(\gamma_{0} \sigma_{e q}\right)^{-4 / 3}$, where $\sigma_{e q}$ is the ratio of Poynting flux to particle kinetic energy output at the equator at the base of the flow and $\gamma_{0}$ the initial Lorentz factor of the flow. The results are applied to the image of the Crab nebula, and the limit is set for the Crab pulsar of $\sigma_{e q} \leq 10^{2}$. It is argued that conventional pulsar theory needs to be reexamined in light of these limits.
\end{abstract}

Subject headings: ISM:jets and outflows—MHD—pulsars:general—stars:winds, outflows - supernova remnants

\section{Introduction}

Magnetic collimation of astrophysical jets was proposed over 20 years ago (Blandford 1976, Lovelace 1976, Benford 1978). Since then, however, there has been considerable debate as to how well it works. Heyvaerts and Norman (1989) and Chiueh, Li, and Begelman (1991) proved that given enough time, all rotating, axisymmetric magnetized flows are collimated by the toroidal magnetic field that is inscribed by the rotation. On the other hand, it was argued (Eichler 1993, Begelman \& Li 1994, Tomimatsu 1994, Bogovalov 1997, 2001, Beskin, Kuznetsova \& Rafikov 1998, Chiueh, Li \& Begelman 1998) that the collimation is so slow (logarithmic) that in practice it could take an exponentially large radius to collimate appreciably, so that all but a small core near the axis would remain uncollimated. Moreover, if the magnetic field dominates the flow, collimation would come at the expense of kink instability. The slowness of the collimation and/or its questionable stability was felt by some to make it unattractive. 
For relativistic outflows with Lorentz factor $\gamma \gg 1$, the problem is particularly severe. Firstly, the radius of curvature of the flow lines scales as $1 / \gamma^{2}$, so that for large enough $\gamma$, the radius of curvature is not only exponentially large, but exponential large with a huge exponent. Secondly, even if the flow started at modest $\gamma$, if it were magnetized, even a small degree of collimation would imply acceleration (Begelman \& Li 1994) to larger $\gamma$, which places even more severe limits on collimation.

However, the remarkable image of the Crab Nebula in the X-rays recently obtained by the CHANDRA Observatory (Weisskopf et al. 2000) convincingly shows a very well defined polar jet. It appears to be a) very well collimated but b) only a small part $\left(\sim 10^{-2}-10^{-1}\right.$ by visual inspection) of the total energy, which is mostly expelled in an apparently equatorial disk. This vindicates both the contentions that a) magnetic collimation can work and that b) it only works on the inner core of an ultra-relativistic outflow. Stability worries may be minimized if most of the jet has been collimated only within its most recent dynamical timescale.

The question is now whether theory can accurately account for the proportions of the polar jet relative to the total pulsar wind, whether the predicted Lorentz factor of the jet is consistent with observational limits on relativistic beaming, and whether the implications of the pulsar wind observations are consistent with the theory of pulsar magnetospheres.

In this paper we consider these questions. We show that the conventional view of the Crab pulsar would allow too miniscule a jet to be consistent with the observed one. We then show that relaxing the usual beliefs about the Crab pulsar and assuming only that the pair luminosity must be less than the gamma ray luminosity allows a jet fraction at most $10^{-2} \gamma_{0}^{-4 / 3}$, just barely consistent with the observations even for $\gamma_{0} \sim 1$. Here $\gamma_{0}$ is the Lorentz factor at the base of the flow. In the following sections, we show that the jet luminosity scales relative to the total as $\left(\gamma_{0} \sigma_{e q}\right)^{-4 / 3}$, where $\sigma_{e q}$ is the ratio of Poynting flux to particle kinetic energy output at the equator at the base of the flow. We note in the discussion section the implications of this for theories of the Crab pulsar.

\section{Basic equations}

We first review the basic theory of relativistic, magnetized winds. At large distances (in fact beyond the fast magnetosonic point) one can consider the flow as a purely poloidal $\left(v_{\varphi}=0\right)$ flow in a purely toroidal magnetic field (cf. Li 1996). Indeed conservation of the angular momentum implies that the azimuthal velocity goes to zero with radius while the contribution of the poloidal field to the force balance becomes eventually negligible because 
the poloidal field falls off faster than the toroidal one. In ultrarelativistic flows, hoop stress and electrical force nearly cancel each other so that the poloidal field pressure, while much less than either term in magnitude, is negligible only far enough from the light cylinder to be smaller than their difference. However, this distance still lies well within the termination shock, and the logarithm of the ratio between the two will be shown to play a role. The momentum conservation equation is given by

$$
\rho(\gamma \mathbf{v} \cdot \nabla) \gamma \mathbf{v}=\rho_{e} \mathbf{E}+\mathbf{j} \times \mathbf{B},
$$

where $\rho$ is the proper mass density, $\mathbf{v}$ the plasma velocity, $\mathbf{E}$ and $\mathbf{B}$ are the electric and magnetic field strengths, correspondingly and $\rho_{e}$ and $\mathbf{j}$ are the charge and current densities, correspondingly. Under the above assumptions one can write

$$
\mathbf{v}=v \mathbf{l} ; \quad \mathbf{B}=B \mathbf{e}_{\varphi} ; \quad \mathbf{E}=-\mathbf{v} \times \mathbf{B}=-v B \mathbf{t} ;
$$

where $\mathbf{l}$ is the longitudinal unit vector along the magnetic surface, $e_{\varphi}$ is the azimuthal unit vector and $\mathbf{t}=\mathbf{l} \times \mathbf{e}_{\varphi}$ is the unit vector in the transverse direction. Ee take the speed of light to be unity. With the Maxwell equations, we can present the current and charge densities in the form

$$
\begin{gathered}
4 \pi \mathbf{j}=\nabla \times \mathbf{B}=\frac{1}{r} \nabla(r B) \times \mathbf{e}_{\varphi} ; \\
4 \pi \rho_{e}=\nabla \cdot[\mathbf{B} \times \mathbf{v}]=-\frac{v}{r} \mathbf{t} \cdot \nabla(r B)-B \mathbf{t} \cdot \nabla v-v B\left(\mathbf{e}_{\varphi} \cdot[\nabla \times \mathbf{l}]\right),
\end{gathered}
$$

where $r$ is the cylindrical radius.

The cross-field equation may be obtained by taking the dot product of Eq.(1) with $\mathbf{t}$ and using the above expressions for $\rho_{e}$ and $j$. After simple algebra, we obtain

$$
\rho\left(\gamma^{2}-1\right) \mathbf{t} \cdot(\mathbf{l} \cdot \nabla) \mathbf{l}=\frac{1}{4 \pi}\left\{v^{2} B^{2}\left(\mathbf{e}_{\varphi} \cdot[\nabla \times \mathbf{l}]\right)-\frac{B}{r \gamma} \mathbf{t} \cdot \nabla \frac{r B}{\gamma}\right\},
$$

where $\gamma=\left(1-v^{2}\right)^{-1 / 2}$ is the flow Lorentz factor. Taking into account the definition of the curvature radius of the flux line

$$
\frac{1}{R_{c}} \equiv \mathbf{t} \cdot(\mathbf{l} \cdot \nabla) \mathbf{l}=-\mathbf{t} \cdot[\mathbf{l} \times[\nabla \times \mathbf{l}]]=-\mathbf{e}_{\varphi} \cdot[\nabla \times \mathbf{l}],
$$

one can write finally

$$
\frac{\gamma^{2}-1}{R_{c}}\left(1+\frac{1}{\sigma}\right)=-\mathbf{t} \cdot \nabla\left(\ln \frac{r B}{\gamma}\right),
$$

where the ratio of the Poynting flux to the kinetic energy flux is

$$
\sigma=\frac{E B}{4 \pi \rho v \gamma^{2}}=\frac{B^{2}}{4 \pi \rho \gamma^{2}} \text {. }
$$


One can see from Eq.(5) that in an ultrarelativistic wind, $\gamma \gg 1$, the flow lines are nearly straight and collimation is possible, if possible at all, only near the axis (Tomimatsu 1994, Chiueh et al. 1998, Bogovalov 2001).

The Lorentz factor of the flow should be determined from the energy conservation along the flux tube. This equation may be obtained by taking the dot product of the momentum equation (1) with the longitudinal vector $\mathbf{l}$. However one can instead simply write down the energy flux per unit poloidal flux

$$
\frac{\rho \gamma^{2} v+\frac{E B}{4 \pi}}{B_{p}}=\text { const. }
$$

Taking into account conservation of the mass flow within the field line tube, we define a quantity $\eta(\Psi)$ that is conserved along each surface $\Psi$ by

$$
\rho v \gamma \equiv \eta(\Psi) B_{p}
$$

Making use of this equation and the relation

$$
E=r \Omega B_{p}
$$

one can write the energy equation as

$$
\gamma+\frac{r^{2} \Omega^{2} B_{p}}{4 \pi \eta(\Psi) v} \equiv \mu(\Psi)
$$

Here the poloidal flux function is defined from the poloidal field strength by

$$
\mathbf{B}_{p}=\frac{1}{r} \nabla \Psi \times \mathbf{e}_{\varphi}
$$

It follows from Eq.(9) that plasma accelerates if $r|\nabla \Psi|$ decreases (Begelman \& Li 1994). In both radial flows and cylindrical ones the Lorentz factor remains constant. (Note that in the case of interest one need not introduce the poloidal field at all but instead define $\Psi$ as a stream function (cf. Contopoulos 1995). We retained the standard definitions to provide a simple comparison with the previous works.)

The asymptotic transfield and energy equations were found by expanding the full set of MHD equations in 1/r by Begelman \& Li (1994), Tomimatsu (1994), Bogovalov (1997, 2001), Chiueh et al (1998), Bogovalov \& Tsinganos (1999). One can show that their equations are equivalent to Eqs. $(5,9)$. Examining the neglected terms one can see that these equations are valid at $r \gg \gamma R_{L}$ where $R_{L}=c / \Omega$ is the light cylinder radius and $\Omega$ the angular velocity of the pulsar. This condition arises because the neglected poloidal field stress $\left(\propto B_{p}^{2} \propto\left(r / R_{L}\right)^{4}\right)$ should be compared in the relativistic case not with the hoop stress $\left(\propto B_{\varphi}^{2} \propto\left(r / R_{L}\right)^{2}\right)$ but with $B_{\varphi}^{2}-E^{2}=B_{\varphi}^{2} / \gamma^{2}$. This condition is equivalent to the condition $R \gg R_{f}$, where $R_{f}$ is the radius of the fast magnetosonic point (Beskin et al. 1998). 


\section{The flow near the axis}

Close enough to the axis, a jet may be formed if flow lines turn through even a small angle $\Delta \theta \sim \theta$. Let us consider the flow near the axis. To specify the conserving fluxes one should know the flow structure in the near zone. Note that at distances exceeding $R_{L}$ the flow fills all the space and plasma density and other parameters vary at the angular scale $\theta \sim 1$. One can naturally assume that close enough to the axis, $\theta \ll 1$, both plasma density and the initial Lorentz factor are close to the values at the axis. Therefore we can take as a good approximation $\eta(\Psi)=$ const and $\gamma_{0}=$ const. The flow lines of the pulsar wind are practically straight inside the fast magnetosonic point (but outside the light cylinder) and the flow is nearly quasiradial in this region. An example of such a flow is provided by the well-known split monopole solution (Michel 1973)

$$
\Psi=\Psi_{e q}(1-\cos \theta) \approx \frac{1}{2} \Psi_{e q} \theta^{2}, \quad B_{\varphi}=\frac{\Psi_{e q} \Omega \sin \theta}{c R}, \quad \mathbf{E}=\frac{\Omega}{c} \nabla \Psi .
$$

Here $R$ and $\theta$ are spherical radius and polar angle, correspondingly, and $\Psi_{e q}$ is the total (equatorial) poloidal flux. This solution is valid within the fast magnetosonic point. Taking into account that $\Psi \propto \theta^{2}$ at $\theta \ll 1$ in any quasiradial flow, one can describe any such a flow near the axis by an equivalent split monopole solution. Note that even in flows that are eventually collimated (e.g., Eichler 1993) the outflows well within the collimation scale reduce to (10), which represents the precollimated state.

The energy integral $\mu$ should in principle be determined by the conditions at the fast magnetosonic point. Careful considerations show that deviations from quasiradial propagation remain small at this point in high $\sigma$ flows (Begelman \& Li 1994, Tomimatsu 1994, Beskin et al 1998). Though these deviations are crusial for determining the position of this point, one can safely neglect them in the angular distribution of the parameters. Substituting equation (10) into equation (9), one gets

$$
\mu=\gamma_{0}+\frac{\Omega^{2} \Psi_{e q} \sin ^{2} \theta}{4 \pi \eta v_{0}} .
$$

Expressing back $\theta$ via $\Psi$, we find the approximate energy integral as

$$
\mu(\Psi)=\gamma_{0}\left\{1+\sigma_{e q}\left(2 \Psi / \Psi_{e q}-\left(\Psi / \Psi_{e q}\right)^{2}\right)\right\} \approx \gamma_{0}\left(1+2 \sigma_{e q} \Psi / \Psi_{e q}\right),
$$

where $\sigma_{e q}$ is the ratio of the Poynting to the matter energy flux at the equator. Because we are only interested in the flow near the axis, the "equatorial" values should be considered not as true values at the equator of the flow but as values at the equator of the split monopole flow that coincides close to the axis with the flow of interest. 
From here on we will use the small angle approximation $\psi \equiv \Psi / \Psi_{e q} \ll 1, r \ll z$ etc. We will consider the flow as ultrarelativistic, $\gamma \gg 1$, because a Poynting dominated flow beyond the fast magnetosonic point is necessarily ultrarelativistic. Note that Poynting flux decreases toward the axis, the initial ratio of the Poynting flux to the kinetic energy flux being

$$
\sigma_{0}=2 \sigma_{e q} \psi \text {. }
$$

Thus, close enough to the axis, $\psi<\left(2 \sigma_{e q}\right)^{-1}$, the flow is matter dominated. This part of the flow is collimated already at the distance $R \sim R_{f}$ (Bogovalov 2001). We are interested in collimation of a wider flux tube where the flow is Poynting dominated.

Note that decreasing of the Poynting flux toward the axis may be understood from a rather simple consideration that demonstrates that this feature is quite general and not a specific feature of Michel's solution. It follows immediately from equation (8) that the electric field decreases toward the axis. The toroidal magnetic field $B_{\varphi} \propto r^{-1} \int j r d r$ also decreases unless the current density $j$ is singular at the axis. Therefore the Poynting flux $E B_{\varphi} / 4 \pi$ decreases toward the axis. Our expression (11) for $\mu(\psi)$ may be considered simply as expansion of general energy flux in small $\psi$.

The shape of the flux surfaces is conveniently described in cylindrical coordinates by the function $r(\psi, z)$ instead of $\psi(r, z)$. Then, e.g.,

$$
B_{p}=\frac{1}{r}|\nabla \psi| \approx \frac{1}{r} \frac{\partial \psi}{\partial r}=\left(r \frac{\partial r}{\partial \psi}\right)^{-1} .
$$

In the small angle approximation the curvature radius may be presented as

$$
R_{c}=\left(\frac{\partial^{2} r}{\partial z^{2}}\right)^{-1}
$$

It follows immediately from Eqs $(6,8)$ that $\sigma \propto r B / \gamma$ when $\eta(\psi)=$ const. Transforming derivatives to independent variables $r$ and $\psi$, one can write Eq.(5) as

$$
\gamma^{2} \frac{\partial r}{\partial \psi} \frac{\partial^{2} r}{\partial z^{2}}=-\frac{\partial \ln (1+\sigma)}{\partial \psi} .
$$

The energy equation (9) is now written, with the aid of Eq.(11), as

$$
\gamma+\frac{\sigma_{e q} \gamma_{0}}{v} r|\nabla \psi|=\gamma_{0}\left(1+2 \sigma_{e q} \psi\right)
$$

Taking into account that $\gamma^{3} \gg \gamma_{0} \sigma_{0}$ beyond the fast magnetosonic point (see, e.g., Beskin et al. 1998), one can substitute $v=1$ into the denominator of the second term in the left-hand 
side of this equation and write finally

$$
\gamma=\gamma_{0}\left[1+\sigma_{e q}\left(2 \psi-r\left(\frac{\partial r}{\partial \psi}\right)^{-1}\right)\right] .
$$

The system of equations is completed by the expression for $\sigma$ (see Eqs. $(6,7,12)$ ):

$$
\sigma=\frac{\Omega^{2} r^{2} B_{p}}{4 \pi \eta \gamma}=\sigma_{e q} r|\nabla \psi| \frac{\gamma_{0}}{\gamma}=\sigma_{e q} r \frac{\gamma_{0}}{\gamma}\left(\frac{\partial r}{\partial \psi}\right)^{-1}
$$

\section{Collimation of the flow}

Bogovalov (2001) very recently showed numerically that in the case of $\gamma_{0}^{2} \leq \sigma_{e q}$ the flow within the tube $\psi<\left(2 \sigma_{e q}\right)^{-1}$ is collimated into a cylindrical jet at the distance of the order of the radius of the fast magnetosonic point. One can see that within this flux tube $\sigma_{0} \leq 1$ and the fraction of the energy carried by the jet is only $\sim \sigma_{e q}^{-2}$. This is insufficient to explain the observed jet in the Crab nebula. Here we consider whether a larger fraction of the poloidal flux (and, correspondingly, of the total energy) may collimate at larger radii.

The initial radial flow (10) may be presented near the axis as $r=\sqrt{2 \psi} z$. Let us look for small deviations from this radial flow,

$$
r=\sqrt{2 \psi} z(1-y) ; \quad y(\psi, z) \ll 1 .
$$

The approximate collimation condition will be defined as follows. We shall solve the equations retaining only terms of the lowest order in $y$ and if we find $y \rightarrow 1$ for some poloidal field line, we conclude that this line collimates.

\subsection{The case $\psi<\left(2 \sigma_{e q}\right)^{-1}$}

It follows from Eq.(11) that under the condition $\psi<\left(2 \sigma_{e q}\right)^{-1}$ the flow is matter dominated. One can approximate this case by assuming $\sigma \ll 1$, which is formally correct for $\psi \ll\left(2 \sigma_{e q}\right)^{-1}$. In this case the flow evidently does not accelerate, $\gamma \approx \gamma_{0}$, and $\sigma$ varies insignificantly, $\sigma \approx \sigma_{0}$, as long as deviation of the flow from the purely radial one is small, $y \ll 1$. Linearizing Eq.(13), one gets with the aid of Eq.(12)

$$
z \gamma_{0}^{2} \frac{\partial^{2}(z y)}{\partial z^{2}}=2 \sigma_{e q}
$$

The solution to this equation is

$$
y=\frac{2 \sigma_{e q}}{\gamma_{0}^{2}} \ln \frac{z}{R_{f}}
$$


The initial point was chosen to be $R_{f}$ because the equations are valid only at $z \gg R_{f}$ and there is no collimation at $z \ll R_{f}$ (Begelman \& Li 1994). One can see that if

$$
2 \sigma_{e q}<\frac{\gamma_{0}^{2}}{\ln \frac{R}{R_{f}}}
$$

where $R$ is the radius of the termination shock, the flow is not collimated at all. If the reverse is true, i.e. if

$$
2 \sigma_{e q}>\frac{\gamma_{0}^{2}}{\ln \frac{R}{R_{f}}},
$$

the flow collimates at $\psi<\left(2 \sigma_{e q}\right)^{-1}$. At $2 \sigma_{e q} \geq \gamma_{0}^{2}$ the flow tube $\psi<\left(2 \sigma_{e q}\right)^{-1}$ collimates already at $z \sim R_{f}$, which agrees with the results by Bogovalov (2001). At the condition (17) more flow than this may collimate but as will be seen below, such additional collimation invariably introduces acceleration, thus invalidating the approximation of the constant $\gamma$.

\subsection{The case $\psi>\left(2 \sigma_{e q}\right)^{-1}$, no acceleration}

In this region we can take $\sigma \gg 1$. Let us first assume that the flow is not accelerated significantly and show that this implies no further collimation. Substituting $\gamma=\gamma_{0}$ and $\sigma=\sigma_{0}$ into Eq.(13) and linearizing it, one gets

$$
z \psi \frac{\partial^{2}(z y)}{\partial z^{2}}=\frac{1}{\gamma_{0}^{2}} .
$$

The solution to this equation is

$$
y=\frac{\ln \frac{z}{R_{f}}}{\gamma_{0}^{2} \psi} .
$$

Because in the region of interest $\sigma_{0} \gg 1$ even a small deviation from the radial flow may result in significant growth of $\gamma$. The linearized energy equation (14) reads

$$
\gamma=\gamma_{0}\left(1-4 \sigma_{e q} \psi^{2} \frac{\partial y}{\partial \psi}\right) .
$$

Our approximation $\gamma \approx \gamma_{0}$ is valid as long as the second term in the brackets remains small as compared with unity. Substituting the solution (19), one can see that our assumption of constant $\gamma$ is already violated at $R \sim R_{f}$ if $4 \sigma_{e q} \geq \gamma_{0}^{2}$; this case will be considered in the next subsection. By Eq.(20) the solution (19) is valid at

$$
4 \frac{\sigma_{e q}}{\gamma_{0}^{2}} \ln \frac{z}{R_{f}}<1 .
$$


If it is valid until the termination shock, $y$ remains less than unity for any $\psi>\left(2 \sigma_{e q}\right)^{-1}$, so we come back to condition (16). If the reverse is true, the acceleration becomes significant at

$$
z_{0}=R_{f} \exp \left(\frac{\gamma_{0}^{2}}{4 \sigma_{e q}}\right)<R
$$

At this point $y \ll 1$. However, beyond this point collimation may ensue. So let us consider collimation in the accelerating flow.

\subsection{The case $\psi>\left(2 \sigma_{e q}\right)^{-1}$, accelerating flow}

Now one can neglect the first term in Eq.(20) and, substituting the Lorentz factor so obtained into Eqs. $(13,15)$ and neglecting the higher order terms in $y$, one obtains

$$
-16 \gamma_{0}^{2} \sigma_{e q}^{2} \psi^{5} z\left(\frac{\partial y}{\partial \psi}\right)^{3} \frac{\partial^{2}(z y)}{\partial z^{2}}=\frac{\partial y}{\partial \psi}+\psi \frac{\partial^{2} y}{\partial \psi^{2}} .
$$

The solution to this equation with logarithmic accuracy (i.e. neglecting terms of $\left[\ln \left(R / R_{f}\right)\right]^{-1}$ ) is

$$
y=\frac{\left((3 / 2) \ln \frac{z}{R_{0}}\right)^{1 / 3}}{2 \psi\left(\gamma_{0} \sigma_{e q}\right)^{2 / 3}} .
$$

Here $R_{0}=R_{f}$ if $4 \sigma_{e q} \geq \gamma_{0}^{2}$ and $R_{0}=z_{0}$ if $\left(\ln \frac{R}{R_{f}}\right)^{-1}<4 \sigma_{e q} / \gamma_{0}^{2}<1$. The fraction of the collimated flux may be estimated from the condition $y \sim 1$ as

$$
\psi_{c}=\frac{\left((3 / 2) \ln \frac{R}{R_{0}}\right)^{1 / 3}}{2\left(\gamma_{0} \sigma_{e q}\right)^{2 / 3}} .
$$

\subsection{Energy considerations}

Substitution of Eq.(24) into Eq.(20) yields

$$
\gamma=2\left(\frac{3}{2} \gamma_{0} \sigma_{e q} \ln \frac{z}{R_{0}}\right)^{1 / 3}
$$

which is consistent with the results by Tomimatsu (1994) and Beskin et al. (1998). In principle, the flow may accelerate up to the Lorentz factor $\gamma_{\max }(\psi)=\gamma_{0} \sigma_{0}=2 \gamma_{0} \sigma_{e q} \psi$. At the collimation point defined from Eq. (24) as a point where $y \sim 1$, one obtains $\gamma \sim \gamma_{\max }(\psi)$. So when a flux line turns through an angle $\Delta \theta \sim \theta$, a significant fraction of the Poynting 
flux is transferred to the plasma and there should be $\sigma \sim 1$ in the collimated part of the flow. The collimation (and acceleration) process has enough time to be nearly completed only within the small fraction (25) of the poloidal flux. Outside this flux tube the flow slowly accelerates according to Eq.(26). However, when the flow enters the termination shock, most of the energy still remains in the form of Poynting flux.

Because Poynting flux decreases toward the axis of the flow, the fraction of the energy carried by the jet is small. Integrating the energy flux (11), one gets $\dot{E}_{j e t} / \dot{E}_{t o t}=1.5 \psi_{c}^{2}$. So the jet carries the fraction of the total energy

$$
\frac{\dot{E}_{j e t}}{\dot{E}_{t o t}}=\frac{3\left((3 / 2) \ln \frac{R}{R_{0}}\right)^{2 / 3}}{8\left(\gamma_{0} \sigma_{e q}\right)^{4 / 3}} .
$$

\section{Discussion}

The fraction of the collimated flux is determined by the parameter $\gamma_{\max }=\gamma_{0} \sigma_{e q}$, which is Michel's (1969) magnetization parameter. This parameter is determined by the amount of plasma emitted by the pulsar. The density of the plasma generated within the pulsar magnetosphere is conveniently normalized by the Goldreich-Julian charge density:

$$
n=\frac{\kappa \rho_{G J}}{e}=\frac{\kappa B \Omega}{2 \pi e c}
$$

where $B$ is the magnetic field in the magnetosphere, $\kappa$ the multiplicity factor. Within the light cylinder the field is nearly dipolar, $B=\mu / R^{3}$, where $\mu$ is the magnetic moment of the star, and conservation of the mass flux implies $n \propto B$. Beyond the light cylinder $n \propto 1 / R^{2}$. The Poynting flux in the pulsar wind may be expressed via the magnetic field at the light cylinder, $B_{L}=\mu \Omega^{3} / c^{3}$, as

$$
W=\frac{c B_{L}^{2}}{4 \pi}\left(\frac{R_{L}}{R}\right)^{2} .
$$

Now one can estimate the magnetization parameter as

$$
\gamma_{\max }=\frac{W}{m n c^{3}}=\frac{e B_{L}}{2 m c \kappa \Omega}=1.3 \times 10^{3} \frac{\mu_{30}}{\kappa_{4} P^{2}},
$$

where $\mu=10^{30} \mu_{30} \mathrm{G} \cdot \mathrm{cm}^{3}, \kappa=10^{4} \kappa_{4}$ and $P$ is in seconds. For the Crab pulsar, where $P=0.033 \mathrm{~s}$ and $\mu_{30}=5$, we have

$$
\gamma_{\max }=\frac{6 \cdot 10^{6}}{\kappa_{4}}
$$

From the observed amount of radio emitting electrons (those with the energy $>100 \mathrm{MeV}$ ) one can get an upper limit for the injection rate of these electrons about $\sim 10^{41} \mathrm{~s}^{-1}$, which 
corresponds to $\kappa \sim 3 \cdot 10^{6}$. If the pulsar ejects a larger amount of pairs, their energy in the nebula should be less than $100 \mathrm{MeV}$.

The standard models of plasma production in pulsars (see, e.g., the review by Arons 1983) typically predict $\kappa \sim 10^{3}-10^{4}$ and $\gamma_{0} \sim 100$. Different modifications were proposed to explain the observed powerful gamma radiation (Cheng, Ho \& Ruderman 1986, Usov \& Melrose 1996, Lyubarskii 1996); these models predict significantly larger plasma production. However, we are not aware of models predicting $\kappa>$ few $\times 10^{6}$. If protons are also emitted by the pulsar, the corresponding magnetization parameter is reduced by a factor $m / m_{p}$. However theoretical arguments (Arons 1983) favor $\kappa_{p} \sim 1$.

It is very difficult to place limits on the wind parameters directly from observations. Limitations found by Wilson \& Rees (1978) who studied induced scattering of the radio emission in the pulsar wind were obtained on the assumption that the cyclotron frequency near the light cylinder is less than the radiation frequency. However the reverse is true in the case of the Crab pulsar (Barnard 1986, Lyubarskii \& Petrova 1996). The scattering rate in the magnetized plasma is small if the pulsar radiates only waves polarized perpendicularly to the local magnetic field (Blandford \& Scharlemann 1976, Lyubarskii \& Petrova 1996). In this case the scattering begins only beyond the polarization limiting radius, which lies, in the Crab case, well outside the light cylinder.

Because pairs are produced by gamma radiation, one can reasonably conclude that the pair luminosity does not exceed the gamma luminosity. This places the limit $\sigma_{e q}>100$. One can see from Eq.(27) that $\dot{E}_{j e t} / \dot{E}_{t o t} \approx 10^{-2}$ even at $\sigma_{e q}=100$ and $\gamma_{0}=1$.

Although the fraction of total luminosity that emanates from the jet is not yet published, and in any case is not quite the same as the fraction of mechanical output that emerges in the jet, visual inspection seems to imply that $10^{-2}$ is a conservative guess. The implication that $\sigma_{e q}$ is at most $10^{2}$ and $\gamma_{0} \sim 1$ runs counter to conventional pulsar wisdom. Probably observed constraints on relativistic beaming will provide an even sharper constraint on $\sigma_{e q}$ and $\gamma_{0}$, making the observed jet even more confusing for conventional pulsar theory. However, since existing theory for pulsar magnetospheres is not rigorous, perhaps it needs to be reexamined. The jet may be formed via hoop stress collimation of the the pulsar wind only if the wind is much more mass-loaded than previously believed. The alternative possibility, that the jet is formed already near or within the pulsar magnetosphere, $R \sim R_{L}$, also requires serious modifications to the conventional picture of the pulsar magnetosphere.

We thank the Israeli Science Foundation and the Arnow Chair of Physics for their generous support. 


\section{REFERENCES}

Arons J., 1983. In: Positron-electron pairs in astrophysics; New York, American Institute of Physics, p. 163-193

Barnard J.J., 1986. ApJ 303, 280

Begelman M. C., Li Z.-Y., 1994. ApJ 426, 269

Benford, G., 1978. MNRAS 183, 29

Beskin V.S., Kuznetsova I.V., Rafikov R.R., 1998. MNRAS 299, 341

Blandford R.D. 1976. MNRAS 176, 465

Blandford R.D. \& Scharlemann E.T. 1974. MNRAS 174, 59

Bogovalov S.V., 1997. A \& A 327, 662

Bogovalov S.V., 2001, A\&A 371, 1155

Bogovalov S.V., Tsinganos K., 1999. MNRAS 305, 211

Cheng T., Ho C. \& Ruderman M. 1986. ApJ 300, 500

Chiueh T., Li Z.-Y., Begelman M.C., 1991. ApJ 337, 462

Chiueh T., Li Z.-Y., Begelman M.C., 1998. ApJ 505, 835

Contopoulos J. 1995. ApJ 450, 616

Eichler D., 1993. ApJ 419, 111

Heyvaerts J. \& Norman, C.A., 1989. ApJ 347, 1055

Li Z.-Y. 1996. ApJ 473, 873

Lovelace R. V. E., 1976. Nature 262, 649

Lyubarskii Y.E., 1996. A\&A 311, 172

Lyubarskii Y.E. \& Petrova S.A. 1996. AstL 22, 399

Michel F.C., 1969. ApJ 158, 727

Michel F.C., 1973. ApJ 180, 133 
Tomimatsu A., 1994, PASJ, 46, 123

Usov V.V. \& Melrose D.B. 1994. ApJ 464, 306

Wilson D.B. \& Rees M.J. 1978. MNRAS 185, 297

Weisskopf C., Hester J.J., Tennant A.F. et al., 2000. ApJ 536, L81 